University of Wollongong

Research Online

Faculty of Engineering and Information

Faculty of Engineering and Information

Sciences - Papers: Part B

Sciences

2019

An approximation formula for the price of credit default swaps under the fast-mean reversion volatility model

Xinjiang He

University of Wollongong, xinjiang@uow.edu.au

Wenting Chen

Jiangnan University, wtchen@uow.edu.au

Follow this and additional works at: https://ro.uow.edu.au/eispapers1

Research Online is the open access institutional repository for the University of Wollongong. For further information contact the UOW Library: research-pubs@uow.edu.au 


\title{
An approximation formula for the price of credit default swaps under the fast- mean reversion volatility model
}

\author{
Abstract \\ We consider the pricing of credit default swaps (CDSs) with the reference asset assumed to follow a \\ geometric Brownian motion with a fast mean-reverting stochastic volatility, which is often observed in the \\ financial market. To establish the pricing mechanics of the CDS, we set up a default model, under which \\ the fair price of the CDS containing the unknown "no default" probability is derived first. It is shown that \\ the "no default" probability is equivalent to the price of a down-and-out binary option written on the same \\ reference asset. Based on the perturbation approach, we obtain an approximated but closed-form pricing \\ formula for the spread of the CDS. It is also shown that the accuracy of our solution is in the order of $O(\varepsilon)$.

\section{Publication Details} \\ He, X. \& Chen, W. (2019). An approximation formula for the price of credit default swaps under the fast- \\ mean reversion volatility model. Applications of Mathematics, 64 (3), 367-382.
}




\title{
AN APPROXIMATION FORMULA FOR THE PRICE OF CREDIT DEFAULT SWAPS UNDER THE FAST-MEAN REVERSION VOLATILITY MODEL
}

\author{
Xin-Jiang He, Wollongong, Wenting Chen, Wuxi Shi
}

Received November 13, 2017. Published online April 9, 2019.

\begin{abstract}
We consider the pricing of credit default swaps (CDSs) with the reference asset assumed to follow a geometric Brownian motion with a fast mean-reverting stochastic volatility, which is often observed in the financial market. To establish the pricing mechanics of the CDS, we set up a default model, under which the fair price of the CDS containing the unknown "no default" probability is derived first. It is shown that the "no default" probability is equivalent to the price of a down-and-out binary option written on the same reference asset. Based on the perturbation approach, we obtain an approximated but closedform pricing formula for the spread of the CDS. It is also shown that the accuracy of our solution is in the order of $\mathscr{O}(\varepsilon)$.
\end{abstract}

Keywords: credit default swaps; fast mean-reverting volatility; perturbation method MSC 2010: 91G20, 91G80

\section{INTRODUCTION}

Credit risk is one of the most important risk types that have been present in finance, commerce and trade transactions ever since the Global Financial Crisis took place in 2008. Due to its harmfulness to the financial market, the effective management of credit risks has become again one of the major topics in today's quantitative finance area and has thus led to the fast development of the credit derivatives. Strictly speaking, credit derivatives are usually classified into two types, single-name and multi-name ones. The former offers protection against credit risk of one particular firm whereas the latter takes into consideration the risks posed by not only individual companies but also correlations between them [2].

This research was supported by National Natural Science Foundation of China Number 11601189, and Natural Science Foundation of Jiangsu Province Number BK20160156. 
One of the most basic single-name credit derivatives is the so-called credit default swaps (CDSs), which is also regarded as the basis of many other credit derivatives. A CDS is an over-the-counter derivative that functions as an insurance contract, which transfers credit risk from one party to another through the mechanics that its buyer makes periodic payments to the seller until the maturity date or a credit event occurs, whereas the seller compensates the buyer in case of default [3].

The credit risks considered in CDS contracts are usually modelled by two kinds of models including the reduced-form models [5], [10], [14] and structural models [13], [12]. The former are mathematically appealing as the probability of default can be extracted from market prices. However, they are unable to capture the wide range of default correlations, as pointed out in [2]. The latter, although complicated in the mathematical sense, use the information from the companies' balance sheets and could thus produce default correlations between different firms. Typical models in this category include the Merton model [13], which characterizes the breach by assuming that the default would occur if the company is insolvent.

In the literature, many attempts have been made for the accurate determination of the price of the CDS. Most work concentrated on pricing CDSs under a "modified" Merton model. By "modified", it is meant that some unrealistic assumptions in this model are removed or at least amended. For example, de Malherbe [4] replaced the geometric Brownian motion used in the Merton model by a Poisson process, and determined the corresponding CDS price by a probabilistic approach. With stochastic intensity models adopted for the default events, Brigo and Chourdakis [2] considered counterparty risk for CDSs in presence of correlation between default of the counterparty and that of the CDS reference credit. Longstaff and Schwartz [12] introduced the stochastic interest rate modelled by a mean-reverting process for the reference asset. Their model was then extended by Zhou [18] by adding a jump component for the asset returns. Cariboni and Schoutens [3] went further and incorporated Lévy processes to model the underlying asset. Recently, He and Chen [9] adopted the generalized mixed fractional Brownian motion in modelling the reference asset and derived a closed-form formula for the prices of CDSs.

In this paper, we consider the valuation of CDSs under a fast mean-reverting stochastic volatility (SV) model introduced in [7]. By "mean-reverting", it refers to a process of getting back to the mean level of its invariant distribution, which is consistent with the results of empirical studies [1]. By "fast", we mean that a short period of time is needed for the SV to reach the level of its long-term mean and the evidence for it was provided by Fouque et al. [7]. Fast mean-reverting is indeed one of the most common features of the volatilities of many kinds of assets [7], [19], and thus, the CDS price would be more accurate when the reference asset is assumed to follow such a process. In addition, in comparison with another recent work regarding 
the pricing of CDSs [3], we assume in our work that the company could default at any time before or at the maturity date, which is a more realistic condition.

Our solution process begins by deriving an analytical expression for the CDS price, in which the "no default" probability still needs to be determined. An equivalence relationship between the unknown probability and a down-and-out binary option is then established. With the perturbation method as used in [7], a sequence of simplified systems governing the price of the down-and-out binary option are obtained and solved. The price of the CDS is then obtained.

The rest of the paper is organized as follows. In Section 2, we introduce the CDS contract and establish the default model. In Section 3, the partial differential equation (PDE) system governing the essential part of CDS prices will be derived and solved. In Section 4, the accuracy of the approximation is theoretically investigated. In Section 5, the accuracy of the approximation is further demonstrated through numerical experiments, and the properties of the new formula are also shown. Concluding remarks are given in the last section.

\section{The Default MOdel}

A CDS is a financial agreement between two parties for the compensation of credit events. The buyer of the CDS should make a series of payments to the seller and, in exchange, receives a payoff if the loan defaults. In the following, the pricing mechanics of a CDS will be presented in details. It should be noted that the price of a swap usually differs from its value. As far as a CDS is concerned, its price is defined as the spread, which is the regular fee that the buyer pays to the seller.

Let $R$ and $M$ be respectively the recovery rate and the face value of the asset, and let $T$ be the expiration time. We assume that both the risk-free interest rate $r$ and the spread $c$ are continuous. To determine the spread $c$, the cash flow of the contract needs to be analyzed first. On one hand, since the CDS buyer should pay the protection fee to the seller only when the default does not occur, we assume that the probability of no-default before the current time $t$ is $p(S, t)$. As a result, the CDS buyer pays $c M \mathrm{~d} t$ to the seller between the times $t-\mathrm{d} t$ and $t$, which implies that the present value of the cash flow of the buyer (denoted by $V_{1}$ ) is

$$
V_{1}=\sum_{t} \mathrm{e}^{-r t} c M p(S, t) \mathrm{d} t=c M \int_{0}^{T} \mathrm{e}^{-r t} p(S, t) \mathrm{d} t .
$$

Since the cash flow of the buyer will not be zero when any reasonable CDS contrast is established, one could conclude that $\int_{0}^{T} \mathrm{e}^{-r t} p(S, t) \mathrm{d} t \neq 0$. On the other hand, the seller of the CDS needs to pay $(1-R) M$ to the buyer only if the reference 
company defaults. Since the default taking place within time $[t, t+\mathrm{d} t]$ would have the probability of

$$
[1-p(S, t+\mathrm{d} t)]-[1-p(S, t)]=p(S, t)-p(S, t+\mathrm{d} t)=-\mathrm{d} p(S, t),
$$

the present value of the cash flow of the seller, denoted by $V_{2}$, can be expressed as

$$
V_{2}=\sum_{t}-\mathrm{e}^{-r t}(1-R) M \mathrm{~d} p(S, t)=-(1-R) M \int_{0}^{T} \mathrm{e}^{-r t} \mathrm{~d} p(S, t) .
$$

Similarly to a forward contract, the CDS should be fair to both parties when it is initiated. Therefore, we have $V_{1}=V_{2}$ implying that

$$
c M \int_{0}^{T} \mathrm{e}^{-r t} p(S, t) \mathrm{d} t=-(1-R) M \int_{0}^{T} \mathrm{e}^{-r t} \mathrm{~d} p(S, t),
$$

from which the price of the CDS can be derived as

$$
\begin{aligned}
c & =\frac{(1-R) \int_{0}^{T}-\mathrm{e}^{-r t} \mathrm{~d} p(S, t)}{\int_{0}^{T} \mathrm{e}^{-r t} p(S, t) \mathrm{d} t}, \\
& =\frac{(1-R)\left[-\left.\mathrm{e}^{-r t} p(S, t)\right|_{0} ^{T}-r \int_{0}^{T} \mathrm{e}^{-r t} p(S, t) \mathrm{d} t\right]}{\int_{0}^{T} \mathrm{e}^{-r t} p(S, t) \mathrm{d} t}, \\
& =\frac{(1-R)\left[1-\mathrm{e}^{-r T} p(S, T)\right]}{\int_{0}^{T} \mathrm{e}^{-r t} p(S, t) \mathrm{d} t}-r(1-R),
\end{aligned}
$$

where the above derivation is based on the fact that $p(S, 0)=1$ and

$$
\int_{0}^{T} \mathrm{e}^{-r t} p(S, t) \mathrm{d} t \neq 0
$$

It should be remarked that the CDS considered in the current work is different from others in the literature [9]. While the latter only allow the default to happen at the maturity date, the former assumes that the company could default at any time before or at the expiration, which is indeed the case in the financial market.

On the other hand, from (2.1) it is clear that the price of the CDS can be determined if $\mathrm{e}^{-r t} p(S, t)$ is known in advance. However, the determination of $p(S, t)$ is never an easy task especially under the fast mean-reverting volatility model currently considered. This issue will be illustrated in detail in the next section. 


\section{Approximation formula for the CDS}

As pointed out in the last section, the price of the CDS can be obtained straightforwardly once the "no default" probability $p(S, t)$ is calculated. In the following, we shall establish the equivalence between $p(S, t)$ and the price of down-and-out binary options, which will then be considered under the fast mean-reverting volatility framework.

3.1. Down-and-out binary options. Recall that $p(S, t)$ is defined as the probability of no default before the current time $t$. Therefore, $p(S, t)$ can be expressed as

$$
p(S, t)=\operatorname{Prob}\left(\min _{0 \leqslant t^{*} \leqslant t} S_{t^{*}}>L\right)=E\left[I_{\left\{\min _{0 \leqslant t^{*} \leqslant t} S_{t^{*}}>L\right\}}\right]
$$

where $S_{t}$ is the price of the reference asset at time $t$, Prob denotes the probability, and $L$ is the default barrier. Moreover, $I$ is the indicator function and $\min _{0 \leqslant t^{*} \leqslant t} S_{t^{*}}>L$ means the smallest value of the underlying over $[0, t]$ is greater than $L$. Consequently, we have

$$
\mathrm{e}^{-r t} p(S, t)=\mathrm{e}^{-r t} E\left[I_{\left\{\min _{0 \leqslant t^{*} \leqslant t} S_{t^{*}}>L\right\}}\right]
$$

where $E$ is the expectation w.r.t. Prob.

Now, denote

$$
P(S, T-t)=\mathrm{e}^{-r t} p(S, t)
$$

We have

$$
P(S, t)=\mathrm{e}^{-r(T-t)} p(S, T-t)=\mathrm{e}^{-r(T-t)} E\left[I_{\left\{\min _{T-t \leqslant \tau^{*} \leqslant T} S_{\left.\tau^{*}>L\right\}}\right.}\right]
$$

from which one can conclude that $P(S, t)$ is in fact the price of a down-and-out binary option written on the asset $S$ with ' $T-t$ ' being the time to maturity and the pay-off function is defined as

$$
h(S)= \begin{cases}1, & \min _{T-t \leqslant \tau^{*} \leqslant T} S_{\tau^{*}}>L, \\ 0, & \text { otherwise. }\end{cases}
$$

At this stage, it is clear that the pricing of the CDS is equivalent to that of a downand-out binary option with barrier $L$. Once the price of the particular barrier option is calculated, the spread of the CDS can be found through (2.1) and (3.1). In the following, we shall concentrate on deriving an approximation formula for the downand-out binary option in a fast mean-reverting volatility framework, and the target CDS price will then be calculated. 
3.2. Pricing system. The fast mean-reverting volatility model is introduced by Fouque et al. in their recent work [7], and is proved to be closer to the real financial market conditions. In this subsection, the pricing of down-and-out binary options will be considered using the SV model, based on which the price of the CDS will be derived.

Assuming that under the martingale measure $\mathbb{Q}$ introduced by Fouque et al. [6], the reference asset $S_{t}$ follows a fast mean-reverting process

$$
\left\{\begin{array}{l}
\frac{\mathrm{d} S_{t}}{S_{t}}=r \mathrm{~d} t+f\left(Y_{t}\right) \mathrm{d} W_{1}^{\mathbb{Q}} \\
\mathrm{d} Y_{t}=\left[\frac{1}{\varepsilon}\left(m-Y_{t}\right)-\frac{\sqrt{2} v}{\sqrt{\varepsilon}} \Lambda\left(Y_{t}\right)\right] \mathrm{d} t+\frac{\sqrt{2} v}{\sqrt{\varepsilon}} \mathrm{d} W_{2}^{\mathbb{Q}} .
\end{array}\right.
$$

Here, $f\left(Y_{t}\right)$ denotes the volatility of the reference asset, with $f(\cdot)$ being a smooth positive function that is both bounded above and bounded away from zero. The quantities $W_{1}$ and $W_{2}$ are two standard Brownian motions correlated with a factor $\varrho \in[-1,1]$. The function $\Lambda(y)$ is the so-called combined risk premium and is defined as

$$
\Lambda(y)=\varrho \frac{\mu-r}{f(y)}+\sqrt{1-\varrho^{2}} \lambda(y)
$$

where $\mu$ and $r$ are the expected return of the asset and the risk-free interest rate, respectively, and $\lambda(y)$ is a bounded function standing for the volatility risk premium. Furthermore, $\varepsilon$ is a positive small parameter and $1 / \varepsilon$ is rate with which the process reverts to its long term mean $m$. The symbol $v^{2}$ represents the variance of the invariant distribution of $Y_{t}$, which determines the long-run level of the volatility fluctuations.

Let $P(S, y, t)$ be the price of the down-and-out binary option with $y$ being the value of $Y$ at time $t$. According to the Feynman-Kac theorem [16], the partial differential equation (PDE) governing $P$ under the fast mean-reverting volatility model can be derived as

$$
\begin{cases}\mathscr{L}^{\varepsilon} P=0, & S>L, y \in(-\infty, \infty), t \in[0, T), \\ P(S, y, T)=1, & S>L, y \in(-\infty, \infty), \\ P(L, y, t)=0, & y \in(-\infty, \infty), t \in[0, T), \\ P(\infty, y, t)=\mathrm{e}^{-r(T-t)}, & y \in(-\infty, \infty), t \in[0, T),\end{cases}
$$

where $\mathscr{L}^{\varepsilon}=\mathscr{L}_{0} / \varepsilon+\mathscr{L}_{1} / \sqrt{\varepsilon}+\mathscr{L}_{2}$, with

$$
\begin{gathered}
\mathscr{L}_{0}=(m-y) \frac{\partial}{\partial y}+v^{2} \frac{\partial^{2}}{\partial y^{2}}, \quad \mathscr{L}_{1}=\sqrt{2} v\left[\varrho S f(y) \frac{\partial^{2}}{\partial S \partial y}-\Lambda(y) \frac{\partial}{\partial y}\right], \\
\mathscr{L}_{2}=\frac{\partial}{\partial t}+\frac{1}{2} f^{2}(y) S^{2} \frac{\partial^{2}}{\partial S^{2}}+r\left[S \frac{\partial}{\partial S}-I\right]
\end{gathered}
$$


$I$ being the identity operator. Similarly to [19], it can be shown that the boundary conditions along the $y$ direction do not need to be imposed when we aim at finding a general solution valid for a wide class of fast mean-reverting SV models. In the following work, we would only consider a solution that satisfies (3.3), but may or may not satisfy the boundary conditions along the $y$ direction if they are required for the well-posedness of (3.3). Financially, the solution is at least valid for volatility levels not being extremely high or low. In fact, the regime under which we analyze the price of the down-and-out binary option is quite meaningful, because in the so-called fast mean-reverting SV scenario, the volatility level fluctuates randomly around its mean level, and the epochs of high or low volatility are relatively short [8].

3.3. Solution process. To find the solution of (3.3), the asymptotic analysis will be adopted. We assume that $P(S, y, t)$ is in the form

$$
P(S, y, t)=\sum_{n=0}^{\infty} \varepsilon^{n / 2} P_{n}(S, y, t),
$$

with appropriate boundness of $P_{n}(n \in N)$ being all included. By substituting (3.4) into the PDE contained in (3.3), we have

$$
\begin{aligned}
\frac{1}{\varepsilon}\left(\mathscr{L}_{0} P_{0}\right) & +\frac{1}{\sqrt{\varepsilon}}\left(\mathscr{L}_{0} P_{1}+\mathscr{L}_{1} P_{0}\right)+\left(\mathscr{L}_{0} P_{2}+\mathscr{L}_{1} P_{1}+\mathscr{L}_{2} P_{0}\right) \\
& +\sqrt{\varepsilon}\left(\mathscr{L}_{0} P_{3}+\mathscr{L}_{1} P_{2}+\mathscr{L}_{2} P_{1}\right)+\mathscr{O}(\varepsilon)=0 .
\end{aligned}
$$

For the lowest order $\mathscr{O}(1 / \varepsilon)$, we obtain

$$
\mathscr{L}_{0} P_{0}=0
$$

Although the expression of $P_{0}$ remains unknown at this stage, one can still conclude that $P_{0}$ does not depend on the variable $y$ and can be expressed as $P_{0}=P_{0}(S, t)$, because the operator $\mathscr{L}_{0}$ is the generator of an ergodic Markov process acting only on $y$.

Now, we turn to the order of $\mathscr{O}(1 / \sqrt{\varepsilon})$. It is obvious that

$$
\mathscr{L}_{0} P_{1}+\mathscr{L}_{1} P_{0}=0
$$

which implies that $\mathscr{L}_{0} P_{1}=0$. Since $\mathscr{L}_{1}$ is again an operator acting only on $y$ and $P_{0}$ has nothing to do with this particular variable, we have $\mathscr{L}_{0} P_{1}=0$. Therefore, $P_{1}$ is also a constant with respect to $y$, i.e., $P_{1}=P_{1}(S, t)$.

Similarly, for the order $\mathscr{O}(1)$, we have

$$
\mathscr{L}_{0} P_{2}+\mathscr{L}_{1} P_{1}+\mathscr{L}_{2} P_{0}=0
$$


which can be further simplified to

$$
\mathscr{L}_{0} P_{2}+\mathscr{L}_{2} P_{0}=0
$$

due to the fact that $P_{1}$ does not depend on $y$. It should also be pointed out that (3.8) is a Poisson equation with respect to $\mathscr{L}_{0}$ in the variable $y$ once $P_{0}$ is calculated. Therefore, according to the Fredholm alternative theorem [15], the solution $P_{2}$ of (3.8) exists if and only if $\mathscr{L}_{2} P_{0}$ is centered with respect to the invariant distribution of $Y_{t}$, i.e.,

$$
\left\langle\mathscr{L}_{2} P_{0}\right\rangle=0
$$

where $\langle\cdot\rangle$ is the inner product defined as $\int_{-\infty}^{\infty} \cdot \varphi(y) \mathrm{d} y$, with $\varphi$ being

$$
\varphi(y)=\frac{1}{v \sqrt{2 \pi}} \mathrm{e}^{-(y-m)^{2} / 2 v^{2}}
$$

Since $P_{0}$ is a constant with respect to $y,(3.9)$ can be written as $\left\langle\mathscr{L}_{2}\right\rangle P_{0}=0$. By taking the boundary conditions into consideration, the PDE system governing $P_{0}$ can be found as

$$
\left\{\begin{array}{l}
\left\langle\mathscr{L}_{2}\right\rangle P_{0}=0, \\
P_{0}(S, T)=1, \\
P_{0}(L, t)=0, \\
\lim _{S \rightarrow \infty} P_{0}(S, t)=\mathrm{e}^{-r(T-t)} .
\end{array}\right.
$$

It is interesting to notice that $\left\langle\mathscr{L}_{2}\right\rangle$ is the Black-Scholes (B-S) operator, $L_{B S}$, with the constant volatility $\sigma$ being replaced by the effective volatility $\bar{\sigma}$ defined as $\bar{\sigma}^{2}=\left\langle f^{2}(y)\right\rangle$. Clearly, $y$ is no longer a variable in $(3.10)$ because the operator $\left\langle\mathscr{L}_{2}\right\rangle$ involves no partial differentiation with respect to $y$ at all. Consequently, $P_{0}(S, t)$ is equal to the down-and-out binary option price in the B-S framework with volatility $\bar{\sigma}$. By using the method of images [11], $P_{0}$ can be found as

$$
P_{0}(S, t)=\mathrm{e}^{-r(T-t)}\left[N\left(d_{1}\right)-\left(\frac{S}{L}\right)^{1-2 r / \bar{\sigma}^{2}} N\left(d_{2}\right)\right]
$$

where $N(\cdot)$ is the standard normal distribution,

$$
d_{1}=\frac{(\ln S-\ln L)+\left(r-\frac{1}{2} \bar{\sigma}^{2}\right)(T-t)}{\bar{\sigma} \sqrt{T-t}},
$$


and

$$
d_{2}=\frac{-(\ln S-\ln L)+\left(r-\frac{1}{2} \bar{\sigma}^{2}\right)(T-t)}{\bar{\sigma} \sqrt{T-t}} .
$$

It should be remarked that the degeneration to the B-S system is indeed expected in the zeroth order and is a reassurance that our solution procedure is correct. Stochastically speaking, when the mean-reversion rate becomes extremely large, the distribution of $Y_{t}$ is the same as its large time distribution, which is almost surely equal to the effective volatility $\bar{\sigma}$. This also explains why the constant volatility in the B-S model should be replaced by $\bar{\sigma}$ in the zeroth-order solution in fast mean-reverting volatility framework.

After $P_{0}$ being solved, we turn to solve for $P_{1}$. The governing equation for $P_{1}$ can be derived by setting the coefficient in front of $\sqrt{\varepsilon}$ to zero, i.e.,

$$
\mathscr{L}_{0} P_{3}+\mathscr{L}_{1} P_{2}+\mathscr{L}_{2} P_{1}=0
$$

which is again a Poisson equation w.r.t. the operator $\mathscr{L}_{0}$ in the variable $y$. Therefore, we have

$$
\left\langle\mathscr{L}_{1} P_{2}+\mathscr{L}_{2} P_{1}\right\rangle=0
$$

which can be re-written as

$$
\left\langle\mathscr{L}_{2}\right\rangle P_{1}=-\left\langle\mathscr{L}_{1} P_{2}\right\rangle
$$

because $P_{1}$ does not depend on $y$ either. From (3.11), it is clear that $\left\langle\mathscr{L}_{1} P_{2}\right\rangle$ needs to be calculated first in order to determine $P_{1}$. According to (3.8) and (3.9), we have

$$
\mathscr{L}_{0} P_{2}=-\mathscr{L}_{2} P_{0}+\left\langle\mathscr{L}_{2} P_{0}\right\rangle=-\frac{1}{2}\left[f^{2}(y)-\bar{\sigma}^{2}\right] S^{2} \frac{\partial^{2} P_{0}}{\partial S^{2}}
$$

from which, $P_{2}$ can be determined as

$$
P_{2}=-\frac{1}{2} \mathscr{L}_{0}^{-1}\left\{\left[f^{2}(y)-\bar{\sigma}^{2}\right] S^{2} \frac{\partial^{2} P_{0}}{\partial S^{2}}\right\}=-\frac{1}{2}[F(y)+c(S, t)] S^{2} \frac{\partial^{2} P_{0}}{\partial S^{2}},
$$

where $\mathscr{L}_{0} F(y)=f^{2}(y)-\bar{\sigma}^{2}$ and $c(S, t)$ is a constant w.r.t. $y$. By substituting the expression of $P_{2}$ back into (3.11), we have

$$
\left\langle\mathscr{L}_{1} P_{2}\right\rangle=-\frac{1}{2}\left\langle\mathscr{L}_{1} F(y)\right\rangle S^{2} \frac{\partial^{2} P_{0}}{\partial S^{2}}=-V_{2} S^{2} \frac{\partial^{2} P_{0}}{\partial S^{2}}-V_{3} S^{3} \frac{\partial^{3} P_{0}}{\partial S^{3}},
$$

where

$$
V_{2}=\frac{v}{\sqrt{2}}\left[2 \varrho\left\langle f F^{\prime}\right\rangle-\left\langle\Lambda F^{\prime}\right\rangle\right]
$$


and

$$
V_{3}=\frac{v \varrho}{\sqrt{2}}\left\langle f F^{\prime}\right\rangle
$$

Therefore, the PDE system for $P_{1}$ is

$$
\left\{\begin{array}{l}
\left\langle\mathscr{L}_{2}\right\rangle P_{1}=Z(S, t), \\
P_{1}(S, T)=0 \\
P_{1}(L, t)=0 \\
P_{1}(\infty, t)=0
\end{array}\right.
$$

where

$$
Z(S, t)=V_{2} S^{2} \frac{\partial^{2} P_{0}}{\partial S^{2}}+V_{3} S^{3} \frac{\partial^{3} P_{0}}{\partial S^{3}}
$$

It can be observed from (3.13) that $P_{1}$ is governed by the same differential operator $\left\langle\mathscr{L}_{2}\right\rangle$ and is a constant w.r.t. $y$ as expected. It should be remarked that the source term contained in (3.13) is from the volatility level correction and the "skew" effect, through which the corrections w.r.t. the fast mean-reverting factor to the zerothorder solutions are made. This PDE system cannot be solved as straightforwardly as we did for the zeroth order solution. In the following, we shall concentrate on solving for (3.13).

The method that will be utilized is mainly based on the properties of the B-S operator, $L_{B S}$. A simple calculation shows that $L_{B S}[(t-T) Z]=Z-(T-t) L_{B S}[Z]=Z$. The last identity is satisfied because

$$
\begin{aligned}
L_{B S}[Z] & =L_{B S}\left[V_{3} S^{3} \frac{\partial^{3} P_{0}}{\partial S^{3}}+V_{2} S^{2} \frac{\partial^{2} P_{0}}{\partial S^{2}}\right] \\
& =V_{3} S^{3} \frac{\partial^{3}}{\partial S^{3}} L_{B S}\left[P_{0}\right]+V_{2} S^{2} \frac{\partial^{2}}{\partial S^{2}} L_{B S}\left[P_{0}\right]=0 .
\end{aligned}
$$

Now, we suppose that the solution of (3.11) can be written as $P_{1}=V(S, t)-(T-t) Z$. It is clear that $V(S, t)$ satisfies

$$
\left\{\begin{array}{l}
\left\langle\mathscr{L}_{2}\right\rangle V=0 \\
V(S, T)=0 \\
V(L, t)=(T-t) Z(L, t) .
\end{array}\right.
$$

The above PDE system can be solved by using the Laplace transform technique, and we found that

$$
\begin{aligned}
V(S, t)=\left(\frac{S}{L}\right)^{1 / 2-r / \bar{\sigma}^{2}} & \frac{\ln S-\ln L}{2 \pi \bar{\sigma}} \int_{t}^{T} \frac{Z(L, T+t-\xi)}{(T-t)^{3 / 2}} \\
& \times \exp \left(-\frac{1}{2}\left(\frac{r}{\bar{\sigma}}+\frac{\bar{\sigma}}{2}\right)^{2}(T-\xi)-\frac{(\ln S-\ln L)^{2}}{2 \bar{\sigma}^{2}(T-\xi)}\right) \mathrm{d} \xi
\end{aligned}
$$


Therefore,

$$
\begin{aligned}
P_{1}= & \left(\frac{S}{L}\right)^{1 / 2-r / \bar{\sigma}^{2}} \frac{\ln S-\ln L}{2 \pi \bar{\sigma}} \int_{t}^{T} \frac{Z(L, T+t-\xi)}{(T-\xi)^{3 / 2}} \\
& \times \exp \left(-\frac{1}{2}\left(\frac{r}{\bar{\sigma}}+\frac{\bar{\sigma}}{2}\right)^{2}(T-\xi)-\frac{(\ln S-\ln L)^{2}}{2 \bar{\sigma}^{2}(T-\xi)}\right) \mathrm{d} \xi-(T-t) Z(S, t) .
\end{aligned}
$$

Practically, one usually stops at the first correction order when a perturbation method is adopted. We shall also stop our process when finding the next order solution, because

$$
\begin{aligned}
\widetilde{P}= & P_{0}+\sqrt{\varepsilon} P_{1}=\mathrm{e}^{-r(T-t)}\left[N\left(d_{1}\right)-N\left(d_{2}\right) \exp \left(-\left(\frac{2 r}{\bar{\sigma}^{2}(z)}-1\right) \ln \frac{S}{L}\right)\right] \\
& +\left(\frac{S}{L}\right)^{1 / 2-r / \bar{\sigma}^{2}} \frac{\ln S-\ln L}{2 \pi \bar{\sigma}} \int_{t}^{T} \frac{\sqrt{\varepsilon} Z(L, T+t-\xi)}{(T-\xi)^{3 / 2}} \\
& \times \exp \left(-\frac{1}{2}\left(\frac{r}{\bar{\sigma}}+\frac{\bar{\sigma}}{2}\right)^{2}(T-\xi)-\frac{(\ln S-\ln L)^{2}}{2 \bar{\sigma}^{2}(T-\xi)}\right) \mathrm{d} \xi-(T-t) \sqrt{\varepsilon} Z(S, t),
\end{aligned}
$$

is already a very good approximation for the price of the binary down-and-out option, bearing in mind that $\varepsilon$ is assumed to be very small. According to the relationship between the price of the CDS and that of the down-and-out binary option, as shown in (2.1), the desired CDS price can be approximated by

$$
c \approx \tilde{c}=\frac{(1-R)\left[1-\left(P_{0}(S, 0)+\sqrt{\varepsilon} P_{1}(S, 0)\right)\right]}{\int_{0}^{T}\left[P_{0}(S, t)+\sqrt{\varepsilon} P_{1}(S, t)\right] \mathrm{d} t}-r(1-R) .
$$

We will show, in the next section, that the accuracy of the approximation is within the order of $\mathscr{O}(\varepsilon)$.

\section{ACCURACy of the approximation}

In this section we discuss the accuracy of the current approximation. There are two main steps involved. We shall first show that $|P-\widetilde{P}| \sim \mathscr{O}(\varepsilon)$, based on which, the accuracy of the approximated CDS price can then be obtained.

Lemma 4.1. For a fixed point $(x, y, t)$ where $t<T$, there exists a constant $C$ such that $|P-\widetilde{P}|<C \varepsilon$.

P r o of. The proof of this lemma is based on the one used in [6]. For simplicity, we only briefly outline the main steps of the proof. Now, we define the higher order approximation $\widehat{P}$ and the residual $R^{\varepsilon}$ as $\widehat{P}=P_{0}+\varepsilon^{1 / 2} P_{1}+\varepsilon P_{2}+\varepsilon^{3 / 2} P_{3}$ 
and $R^{\varepsilon}=\widehat{P}-P$, respectively, where $P_{1}, P_{2}$ and $P_{3}$ are the same as defined in the previous sections. After some algebraic manipulations, we find that $R^{\varepsilon}$ satisfies $\mathscr{L}^{\varepsilon} R^{\varepsilon}=\varepsilon G_{1}+\varepsilon^{3 / 2} G_{2}$, where $G_{1}(S, y, t)=\mathscr{L}_{2} P_{2}+\mathscr{L}_{1} P_{3}$ and $G_{2}(S, y, t)=\mathscr{L}_{2} P_{3}$. At the expiration date $T$, we have $R^{\varepsilon}(S, y, T)=\varepsilon P_{2}(S, y, T)+\varepsilon^{3 / 2} P_{3}(S, y, T)$. Taking other boundary conditions into consideration, we find that $R^{\varepsilon}$ can be expressed in the following expectation form:

$$
\begin{aligned}
R^{\varepsilon}=\varepsilon E\left\{\left[\mathrm{e}^{-r(T-t)} P_{2}\left(S_{T}, Y_{T}, T\right)\right.\right. \\
\left.\quad-\int_{t}^{T} \mathrm{e}^{-r(T-s)} G_{1}\left(S_{s}, Y_{s}, s\right) \mathrm{d} s\right] I_{\left\{\min _{0 \leqslant t^{*} \leqslant t} S_{\left.t^{*} \geqslant L\right\}} \mid S_{t}, Y_{t}\right\}} \\
+\varepsilon^{3 / 2} E\left\{\left[\mathrm{e}^{-r(T-t)} P_{3}\left(S_{T}, Y_{T}, T\right)\right.\right. \\
\left.\left.\quad-\int_{t}^{T} \mathrm{e}^{-r(T-s)} G_{2}\left(S_{s}, Y_{s}, s\right) \mathrm{d} s\right] I_{\left\{\min _{0 \leqslant t^{*} \leqslant t} S_{t^{*}} \geqslant L\right\}} \mid S_{t}, Y_{t}\right\}
\end{aligned}
$$

where $E$ is the expectation under the measure $\mathbb{Q}$. Expanding $G_{2}$ and $G_{3}$, one can find that they are indeed linear combinations of $S^{n} \partial P_{0}^{n} / \partial S^{n}$. Based on the expression of $P_{0}$ and the technique used in [6], one can show that the expectations appearing in the above expression are bounded by some constants which may depend on $S, y$ and $t$. Therefore, we have

$$
|P-\widetilde{P}| \leqslant|P-\widehat{P}|+|\widehat{P}-\widetilde{P}| \leqslant C \varepsilon,
$$

where $C$ is a constant. This completes the proof of this lemma.

Theorem 4.1. Assuming that all the parameters are in the order of $\mathscr{O}(1)$, we have $|c-\tilde{c}| \sim \mathscr{O}(\varepsilon)$.

Pro of. Due to Lemma 4.1 and the fact that $P$ is the price of a down-and-out binary option and is thus bounded by 0 and $\mathrm{e}^{-r(T-t)}$, we find that

$$
\begin{aligned}
|c-\tilde{c}| & =(1-R)\left|\frac{1-\widetilde{P}(S, y, 0)}{\int_{0}^{T} \widetilde{P}(S, y, t) \mathrm{d} t}-\frac{1-P(S, y, 0)}{\int_{0}^{T} P(S, y, t) \mathrm{d} t}\right| \\
& =(1-R) \frac{\left|\int_{0}^{T}(P+\mathscr{O}(\varepsilon)) \mathrm{d} t[1-P(S, y, 0)]-\int_{0}^{T} P \mathrm{~d} t[1-P(S, y, 0)-\mathscr{O}(\varepsilon)]\right|}{\left|\int_{0}^{T}(P+\mathscr{O}(\varepsilon)) \mathrm{d} t \int_{0}^{T} P \mathrm{~d} t\right|} \\
& \leqslant \mathscr{O}(\varepsilon) \frac{\left|\int_{0}^{T} P \mathrm{~d} t-T[1-P(S, y, 0)]\right|}{\left(\int_{0}^{T} P \mathrm{~d} t\right)^{2}} \sim \mathscr{O}(\varepsilon) .
\end{aligned}
$$

This completes the proof of this theorem. 


\section{Numerical EXAMPles AND Discussions}

At the end of the last section, it is theoretically shown that the current formula is accurate to the order of $\varepsilon$. To numerically verify this issue, in this section, numerical experiments are carried out to test the accuracy of this formula. Furthermore, the impact of the introduction of the fast mean reversion volatility on the CDS price is also discussed through the comparison of the current formula and the corresponding one under the classical Black-Scholes (B-S) model.

To facilitate the numerical computation, it is better to choose a particular SV model, where the parameters appearing in our approximation formula can be explicitly worked out. In our analysis shown in this section, the model used in [6] is adopted, where $f(y)$ and $\lambda(y)$ are set to $\mathrm{e}^{y}$ and 0 , respectively. Under this special SV model, the explicit forms of the parameters needed in our approximation are: $\bar{\sigma}^{2}=\exp \left(2 v^{2}+2 m\right), V_{3}=\sqrt{2} \varrho \exp (5 v+3 m)\left(1-\exp \left(2 v^{2}\right)\right) /(2 v)$, and $V_{2}=\sqrt{2} \varrho(\mu-r) \exp (v+m)\left(-1+\exp \left(2 v^{2}\right)\right) /(2 v)+2 V_{3}$. In addition, unless otherwise stated, the parameters used in this section are listed as follows. The expected return of the reference asset price $u$ is set to 0.2 , while the risk-free interest rate takes the value of 0.04 . In addition, $m$, the long term mean of the volatility, and $v$, the volatility of the invariant distribution of $Y_{t}$, are respectively allocated as $\ln (0.1)$ and $1 / \sqrt{2}$, and the correlation $\varrho$ between the reference asset price and the volatility is -0.2 . We also have the expiry time $T=1$, the current time $t=0$, the spot reference asset price $S_{0}=11$, the default barrier $L=10$, the recovery rate $R=50 \%$ and the positive small parameter controlling the speed of the mean reversion $\varepsilon=1 / 200$.

Before the model comparison is made, it is necessary for us to check whether our approximation has a reasonable degree of accuracy. Due to the fact that the approximation is only made by determining the no-default probability, it suffices for us to compare the no-default probability calculated through our formula and the one obtained through the Monte-Carlo simulation. The results are presented in Figure 1, where the no-default probability under both the approaches shows a downward trend with respect to the time to expiry. This is indeed reasonable because an increase in the time to expiry will lead to a higher chance for the reference asset price to drop below the default level. On the other hand, from this figure, it is also clear that our no-default probability is point-wisely close to the corresponding one calculated by

the Monte-Carlo simulation, with the maximum relative difference between the two being less than $2 \%$. This clearly demonstrates the accuracy of our approximation.

With confidence in the correctness of our formula, we now turn to study the influence of introducing the fast mean-reversion SV on the CDS price. Since our model is in fact a SV correction to the B-S model, the CDS prices under these two models are plotted and compared to further illustrate the impact of the introduction 


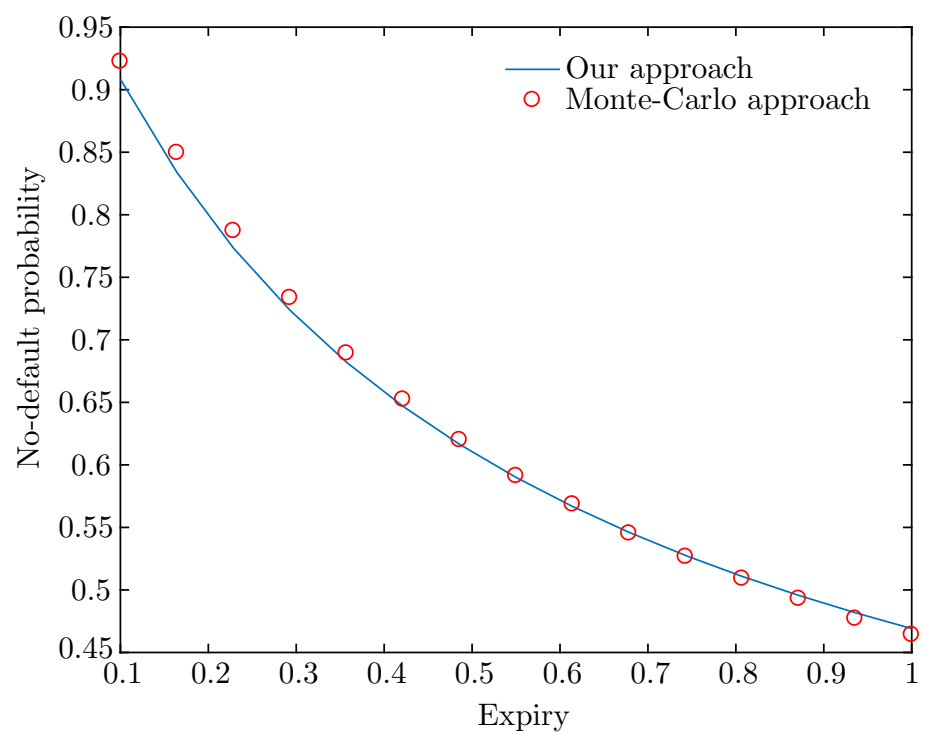

Figure 1. The comparison of the no-default probability calculated from our formula and the Monte-Carlo simulation.

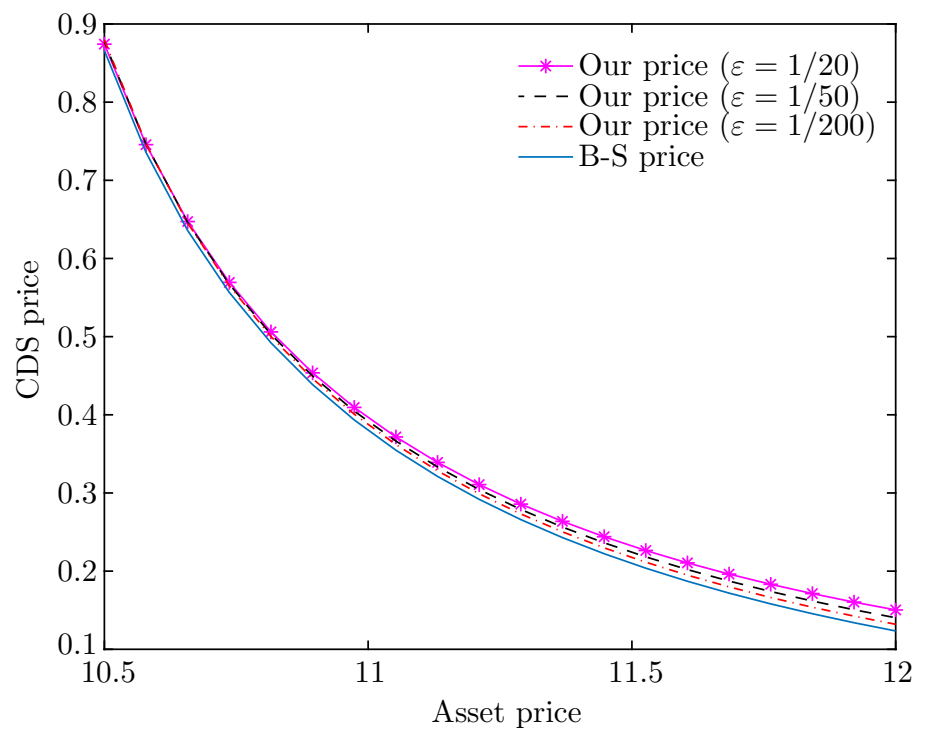

Figure 2. Our price vs B-S price.

of the fast mean-reversion SV, as shown in Figure 2. Note that in this figure, the B-S price is produced with the volatility being equal to $\bar{\sigma}$. From this figure, one can clearly observe that the CDS price is a monotonic decreasing function of the reference asset price. This could be explained by the fact that there will be a lower 
probability for the default to take place when the reference asset price becomes higher. Moreover, one can also observe that the B-S price is always lower than our price. This is consistent with the financial intuition that the introduction of the stochastic volatility makes the reference asset price more volatile, leading to a higher risk and thus a higher price for the derivative. It is also interesting to notice that the difference between our price and the B-S price decreases as the mean-reversion speed increases. A possible explanation for this is that when the mean-reversion speed becomes larger, the SV could be on its "effective" level, and would then behave similarly as the constant one does.

It needs to be remarked that our analytical approximation will have other two main applications in practice. First, our approximation is able to produce a reasonably accurate CDS price in a shorter time, implying that considerable amount of time and effort could be saved in terms of calibration when the fast mean-reversion SV model is applied to real financial markets. The second application of our approximation is that we are now able to compute the analytical approximation of the hedging parameters (or the Greeks), including $\Delta, \Gamma, \theta$, Vega and Rho. This could be achieved by simply differentiating our formula (3.17) with respect to the corresponding variable, and the obtained analytical approximations for these important hedging parameters are certainly very useful in real markets; if they were to be computed based on the numerical solution of the CDS prices, there can be very large errors if the basis functions adopted in the numerical procedure do not have a sufficient degree of differentiability [17]. The final expressions of the hedging parameters are omitted here, because the differentiation process can be somewhat tedious and their expressions would be quite lengthy due to the convoluted expression of our pricing formula (3.17). However, it should still be noted that this is actually a very trivial task nowadays, especially due to the availability of symbolic calculation packages in many programming softwares.

\section{Conclusion}

In this paper, the pricing of the CDS is investigated under the fast-mean reverting SV model. The analytical expression of the price of the CDS, which contains the unknown "no default" probability, is derived first. It is then shown that the discount "no default" probability is equivalent to the price of a down-and-out binary option. By using the method of asymptotic analysis, we found an $\mathscr{O}(\varepsilon)$ approximation formula for the price of this particular option, and also for the price of the corresponding CDS. 


\section{References}

[1] S. Beckers: Variances of security price returns based on high, low, and closing prices. J. Business 56 (1983), 97-112.

[2] D. Brigo, K. Chourdakis: Counterparty risk for credit default swaps: impact of spread volatility and default correlation. Int. J. Theor. Appl. Finance 12 (2009), 1007-1026.

[3] J. Cariboni, W. Schoutens: Pricing credit default swaps under Lévy models. J. Comput. Finance 10 (2007), 71-91.

zbl MR doi

E. De Malherbe: A simple probabilistic approach to the pricing of credit default swap covenants. J. Risk 8 (2006), 85-113.

[5] D. Duffie, K. J. Singleton: An econometric model of the term structure of interest-rate swap yields. J. Finance 52 (1997), 1287-1321.

[6] J.-P.Fouque, G.Papanicolaou, K.R. Sircar: Derivatives in Financial Markets with Stochastic Volatility, Cambridge University Press, Cambridge. 2000.

7] J.-P. Fouque, G. Papanicolaou, K. R. Sircar: Mean-reverting stochastic volatility. Int. J. Theor. Appl. Finance 3 (2000), 101-142.

8] J.-P. Fouque, G. Papanicolaou, K. R. Sircar, K. Solna: Singular perturbations in option pricing. SIAM J. Appl. Math. 63 (2003), 1648-1665.

9] X. He, W. Chen: The pricing of credit default swaps under a generalized mixed fractional Brownian motion. Physica A 404 (2014), 26-33.

[10] R. A. Jarrow, S. M. Turnbull: Pricing derivatives on financial securities subject to credit risk. J. Finance 50 (1995), 53-86.

[11] R. C. Joy, E. S. Schlig: Thermal properties of very fast transistors. IEEE Transactions on Electron Devices 17 (1970), 586-594.

[12] F. A. Longstaff, E.S.Schwartz: A simple approach to valuing risky fixed and floating rate debt. J. Finance 50 (1995), 789-819.

[13] R. C. Merton: On the pricing of corporate debt: the risk structure of interest rates. J. Finance 29 (1974), 449-470.

[14] D. O'Kane, S. Turnbull: Valuation of credit default swaps. QCR Quarterly 2003-Q1/Q2 (2003), 17 pages.

[15] A. G. Ramm: A simple proof of the Fredholm alternative and a characterization of the Fredholm operators. Am. Math. Mon. 108 (2001), 855-860.

[16] S. E. Shreve: Stochastic Calculus for Finance II. Continuous-Time Models. Springer Finance, Springer, New York, 2004.

[17] D. Tavella, C. Randall: Pricing Financial Instruments: The Tinite Difference Method. John Wiley \& Sons, New York, 2000.

[18] C. Zhou: The term structure of credit spreads with jump risk. J. Banking \& Finance 25 (2005), 2015-2040.

zbl MR

19] S.-P. Zhu, W.-T. Chen: Pricing perpetual American options under a stochastic-volatility model with fast mean reversion. Appl. Math. Lett. 24 (2011), 1663-1669.

Authors' addresses: Xin-Jiang He, School of Mathematics and Applied Statistics, University of Wollongong, Northfields Ave, Wollongong NSW 2522, Australia; Wenting Chen (corresponding author), School of Business, Jiangnan University, Liangjiang Rd, Binhu Qu, Wuxi Shi, Jiangsu Sheng, China, e-mail: cwtwxuow@gmail.com. 